\title{
Perceptions of Risk of Health Disparities amid Previously Identified Political Corruption and Ageism in Slovakia
}

\author{
Marta N. Lukacovic \\ Furman University \\ Greenville, SC, USA \\ Deborah D. Sellnow-Richmond \\ Southern Illinois University Edwardsville \\ Edwardsville, IL, USA \\ Monika Durechova \\ Independent Contractor \\ Rajec, SLOVAKIA
}

\begin{abstract}
This study examines the role of traditional versus new media's impact on citizens' perceptions of risk within elder care. We analyzed survey data from 112 Slovak citizens regarding their social network activity and perceptions of corruption, ageism, and health disparities in the Slovak elder care system. Previous research (Lukacovic, SellnowRichmond, \& Durechova, under contract) identified three prominent sociopolitical issues present in the Slovak medical system among Slovak UGC discourse regarding Slovak healthcare inequity: corruption, threats to dignity, and discrimination. Here, we examined the extent to which social media users perceive the prevalence and subsequent risk of experiencing health disparities within the elder care system differently than traditional media users, as well as the extent to which perceived risks of health disparities and mistreatment are stronger among women participants than men.
\end{abstract}

Keywords - Ageism, health disparities, political corruption, risk perception

SUGGESTED CITATION: Lukacovic, M. N., Sellnow-Richmond, D. D., \& Durechova, M. (2019). Perceptions of risk of health disparities amid previously identified political corruption and ageism in Slovakia. Proceedings of the International Crisis and Risk Communication Conference, Volume 2 (pp. 47-50). Orlando Fl: Nicholson School of Communication and Media. https://doi.org/10.30658/icrcc.2019.14

\section{INTRODUCTION}

Previous research [1] identified three prominent sociopolitical issues present in the Slovak medical system among Slovak UGC (user-generated content) discourse regarding Slovak healthcare inequity: corruption, threats to dignity, and discrimination. In this study, we examine the role of traditional versus new media's impact on citizens' perceptions of risk within elder care. We analyze survey data collected in Summer 2017 from 112 Slovak citizens regarding their social network activity and perceptions of corruption, ageism, and health disparities in the Slovak elder care system. The study provides a venue for interdisciplinary connection, as Deegan-Krause [2] stated that corruption is a pressing and prevalent political problem in Slovakia. Hence, such broad problem is epitomized on multiple levels, which require interdisciplinary scholarly investigations.

\section{LITERAURE REVIEW}

Corruption within the Slovak medical system has been addressed by both news organizations and internet users, which was the focus of previous research [1]. Niederdeppe, Bigman, Gonzales and Gollust (2013) argued that mass mediated digital technology for communication is well-suited for conversations about health disparities and can lead to policy and social change. They suggest that localized groups have the potential to take advantage of social media "to shape 
discourse about health disparities" (p. 19) [3]. These projects can give voice to and increase the visibility of those underserved populations hit hardest by health disparities, providing a space to engage in personal storytelling and spur public awareness and action. Therefore, it is worthwhile to consider Slovak internet use in the context of perceived likelihood that they will encounter corruption in the medical system as they age.

The proliferation of new media technologies significantly impacted the accessibility of creating and disseminating UGC. Van Dijck (2009) defined UGC as produced by "active Internet contributors, who put in a 'certain amount of creative effort' which is 'created outside of professional routines and platforms"” (p. 41) [4]. The recent report of the Freedom House stated that $75 \%$ of households in Slovakia have access to broadband Internet connection and $79 \%$ of the population is considered "digitally literate" [5]. This trend predicts these numbers are to increase in future. Essentially, the Internet is the space where a large portion of population interacts. Throughout the year 2014 and at the beginning of the year 2015, a number of incidents suspecting malpractice, due to the existing disparities, have been brought to the attention of mainstream mass media outlets. While the mainstream media paid limited attention to the cases, the discussion about the problems in the Slovak healthcare system was furthered on various Internet platforms in the form of UGC. Hence, this material provides an interesting foundation for analysis in order to explore Slovak citizens' perceptions of health disparities. We consider Beaunoyer, Arsenault, Lomanowska, and Gjuitton's (2017) argument that "online applications where users have a more active role, such as social media, permit the introduction of additional informational elements" including the introduction of "emotional content that may impact the subsequent interpretation of the content by the users" (p. 185) [6].

Marshall (2007) advocated for the study of discrimination based on ageism as an oft overlooked social issue [7]. We examine the extent to which social media users perceive the prevalence and subsequent risk of experiencing health disparities within the elder care system more than traditional media users. Moreover, mid-life women often experience greater pressure than men to reduce paid work in order to care for a frail or elderly parent [8]. Based on this literature, the following two research questions were offered:

1. To what extent do social media users perceive the prevalence and subsequent risk of experiencing health disparities within the elder care and health care system?

2. To what extent are perceived risk of health disparities and mistreatment stronger among women than men?

\section{METHOD}

\section{SAMPLE AND PROCEDURE}

The variable relationships proposed above are explored based on survey data. The research participants were recruited during June, July, and August of 2017 using convenience sampling and subsequent snowball sampling techniques in various locations in Slovakia. Preliminary results show that 138 potential participants were approached, while 112 individuals filled out the questionnaire ( $81 \%$ response rate). Six out of Slovakia's eight main regions were represented in the sample. The mean age of the participants was 42 years $(\mathrm{SD}=16)$, with a range of 18 to 79 years. 59\% of participants were women. $37 \%$ of participants reported college education, while $57 \%$ reported high school and $5 \%$ of participants reported less than a high school level of education.

\section{MEASURES}

The survey questionnaire was used to measure the variables in the current study. The dependent variable (DV) in this study is perceived risk of experiencing disadvantages and disparities within the healthcare system due to either age or socio-economic status (SES). Each of the two types of perceived risk was measured by corresponding survey question, with close-ended responses on 6-point scale ranging from "never" $(M=0)$ to "always" $(M=5)$. The first independent variable (IV 1) is social media use. Two nominal levels of the variable were determined, which are high social media use (visiting social media sites few times a week or more) and low social media use (visiting social media sites few times a month or less). The second independent variable (IV 2) is gender. It was measured as two possible nominal levels, female or male.

\section{RESULTS}

Analysis of variance (ANOVA) using SPSS software was performed to explore the research questions. The test demonstrated that there was not a significant difference in the perception of risk of health disparities due to patient's age when comparing groups based on high and low use of social media, $\mathrm{F}(1,109)=.090, \mathrm{p}=.764$, and gender, $\mathrm{F}(1,109)=$ $.065, \mathrm{p}=.799$. Interaction effect was also insignificant, $\mathrm{F}(1,109)=2.962, \mathrm{p}=.088$. The results for compared groups were; women with low social media use $(\mathrm{M}=3.42, \mathrm{SD}=1.54)$, women with high social media use $(\mathrm{M}=3.07, \mathrm{SD}=$ $1.44)$, men with low social media use $(\mathrm{M}=3.15, \mathrm{SD}=1.69)$, and men with high social media use $(\mathrm{M}=3.56, \mathrm{SD}=1.73)$.

Importantly, a significant difference was detected when comparing perceptions of risk of health disparities due to patient's SES, based on interaction between social media use and gender, $\mathrm{F}(1,109)=4.696, \mathrm{p}=.033$. However, individual effects of social media use, $F(1,109)=1.736, p=.191$, and gender, $F(1,109)=.033, p=.857$, on perceptions of risk of health disparities due to patient's SES were insignificant. The results for compared groups were; 
women with low social media use $(\mathrm{M}=3.32, \mathrm{SD}=1.60)$, women with high social media use $(\mathrm{M}=3.15, \mathrm{SD}=1.40)$, men with low social media use $(\mathrm{M}=2.90, \mathrm{SD}=1.48)$, and men with high social media use $(\mathrm{M}=3.76, \mathrm{SD}=1.36)$ see Figure below.

\section{Perception of the Risk of Health Disparities Due to Socio-Economic Status}

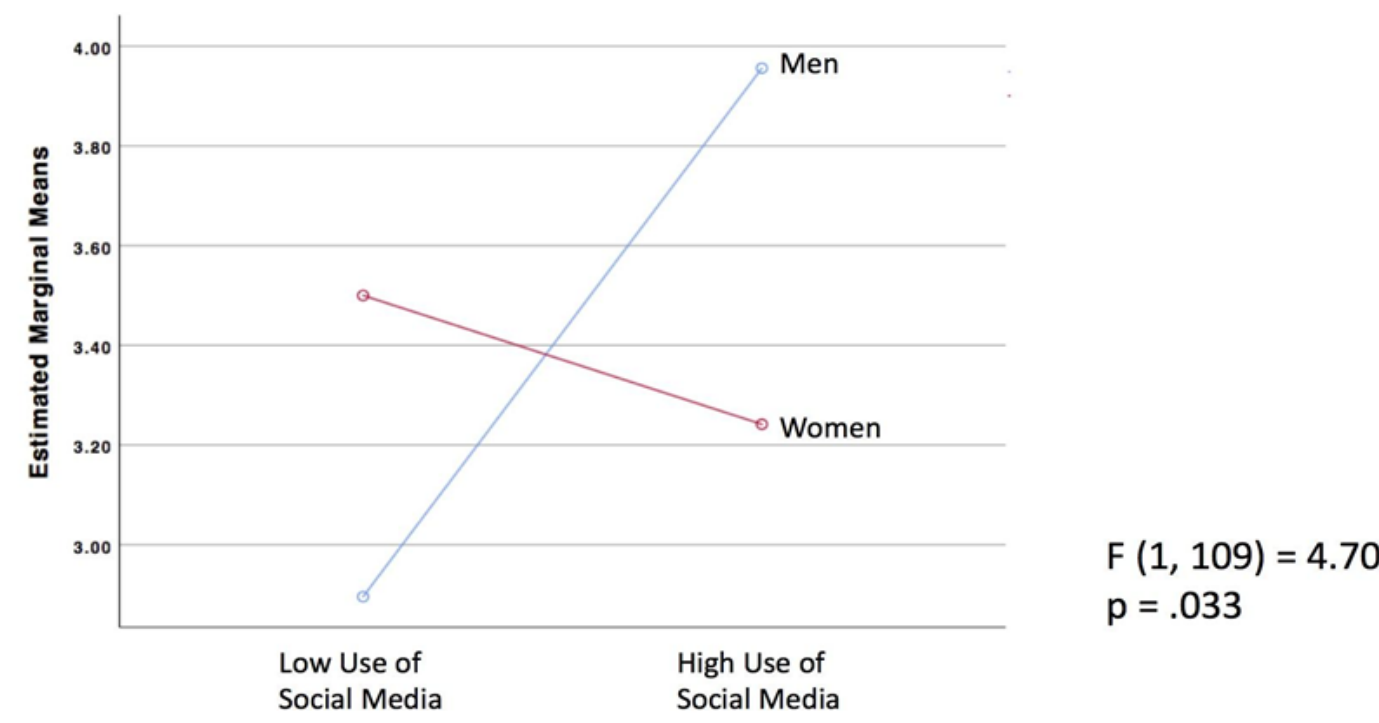

\section{CONCLUSION}

Results of this study indicated overall high perceptions of risk of health disparities. This reflects previous findings [1] that revealed concerns of corruption and threats to dignity within the healthcare system among Slovak internet users. Among participants, vast majority indicated that health disparities and mistreatment of patients due to ageism or SES occur either "most of the times" or "almost always."

Interestingly, some differences in the perceptions are associated with social media use and gender. Specifically, men who reported low use of social media indicated relatively lower risk perceptions regarding the care for lower SES individuals. However, this perception rose considerably among men who reported high use of social media. In contrast, women with lower social media use indicated higher (though not significantly) perceptions of this risk, and the perceived risk decreased as reported social media use increased. This likely reflects the type and purposes of social media women choose to consume versus men. Yet, the findings do not provide evidence of substantially different perceptions of risk by women in comparison to men across conditions. These findings are in contrast to research that suggests elder care is often gendered and more likely to impact women than men. Perhaps the equivalent perceptions of risk among women and men relate to the general distrust to the institutions of health care and other governmental institutions that are responsible for monitoring structural issues and addressing multiple cases of bribery, malpractice, mistreatment, and discriminations based on ageism and other factors.

Several practical recommendations can be offered. First, medical cases should be investigated and rectified by the responsible institutions. Second, policy should respond to structural problems within the Slovak medical system, rather than simply prosecuting individual corrupt actors. Third, institutions should communicate responses to the cases as well as policy changes. Our findings indicate that this should be communicated through traditional and new media channels in order to reach the largest segment of the population. This transparency moving forward has the power to reduce perceived risk of mistreatment within the elder care system. Fourth, patient experiences and public opinion should be continuously monitored. As the population has demonstrated concern over the corruption of the health and elder care system, policy should reflect investment in rectifying previous errs. Engaging the population to gauge shifts in public opinion can help direct the reflective action of policy institutions. Finally, responsible institutions should be attentive to the data on patient experiences and public opinions. The public's primary interaction with the healthcare system is through their own lived experiences. If these institutions effectively understand and respond to public concerns, they can contribute to shifting the negative perceptions surrounding corruption within their system by demonstrating equal, positive treatment of patients. 


\section{Author Biography}

Marta N. Lukacovic, Ph.D. (Wayne State University) is an assistant professor of media and digital communication at Furman University. She can be reached at marta.lukacovic@furman.edu

Deborah D. Sellnow-Richmond, Ph.D. (Wayne State University) is an assistant professor of strategic communication at Southern Illinois University Edwardsville.

Monika Durechova, J.D. (independent contractor; activist) is founder and president of a non-profit organization Slovenska Asociacia Zien (Slovak Association of Women).

\section{REFERENCES}

[1] Lukacovic, M. N., Sellnow-Richmond, D. D., \& Durechova, M. (under contract). User-generated content on health disparities in Slovakia. In Minielli, M., Finch, M., Lukacovic, M., \& Samoilenko, S. (Eds.), Traditional and New Media Studies in Eastern Europe and Eurasia. Lanham, MD: Lexington Books.

[2] Deegan-Krause, K. (2013). Slovakia. In S. Berglund, J. Ekman, K. Deegan-Krause, \& T. Knutsen (Eds.), The handbook of political change in Eastern Europe (3rd ed.) (pp. 255-290), Cheltenham, UK: Edward Elgar.

[3] Niederdeppe, J., Bigman, C. A., Gonzales, A. L., \& Gollust, S. E. (2013). Communication about health disparities in the mass media. Journal of Communication, 63, 8-30. https://doi.org/10.1111/jcom.12003

[4] Van Dijck, J. (2009). Users like you? Theorizing agency in user generated content. Media, Culture, \& Society, 31, 41-58. https://doi.org/10.1177/2F0163443708098245

[5] Meseznikov, G., Kollar, M., \& Butora, M. (2014). Slovakia. Nations in transit 2014 (pp. 563-584). Retrieved from Freedom House website: http://freedomhouse.org/report/nations-transit/2014/slovakia\#.VFFO_pUtBMs

[6] Beaunoyer, E., Arsenault, M., Lomanowska, A. M., \& Guitton, M. J. (2017). Understanding online health information: Evaluation, tools, and strategies. Patient Education and Counseling, 100, 183-189. https://doi.org/10.1016/j.pec.2016.08.028

[7] Marshal, V. W. (2007). Advancing the sociology of ageism. Social Forces, 86, 257-264. https://doi.org/10.1353/sof.2007.0104

[8] Naldini, M., Pavolini, E., \& Solera, C. (2016). Female employment and elderly care: the role of care policies and culture in 21 European countries. Work, Employment and Society, 30, 607-630. https://doi.org/10.1177/0950017015625602 